\title{
Immuno-Chemotherapy Using Repeated Vaccine Treatment Can Produce Successful Clinical Responses in Advanced Metastatic Melanoma
}

\author{
Brendon J. Coventry ${ }^{1}$, Peter Hersey ${ }^{2}$, Anne-Marie Halligan ${ }^{1}$, Antonio Michele ${ }^{3}$ \\ ${ }^{1}$ Discipline of Surgery, University of Adelaide, Royal Adelaide Hospital, Adelaide, Australia; ${ }^{2}$ Newcastle Melanoma Unit, Calvary \\ Mater Newcastle and Medical Oncology and Immunology Unit, University of Newcastle, New South Wales, Australia; ${ }^{3}$ Medical \\ Oncology, North Adelaide Oncology, Calvary Hospital, North Adelaide, Australia. \\ Email: brendon.coventry@adelaide.edu.au, Peter.Hersey@newcastle.edu.au, T.michele@naonc.com.au, Anne-Marie.Halligan \\ @health.sa.gov.au
}

Received August $16^{\text {th }}, 2010$; revised September $10^{\text {th }}, 2010$; accepted $17^{\text {th }}, 2010$.

\begin{abstract}
Advanced Stage IV and IIIc melanoma has a dismal survival, with or without, standard chemotherapy. New therapies are required to improve survival and reduce morbidity. Repeated vaccine dosing does not appear to have been explored, so Vaccinia Melanoma Cell Lysate (VMCL) vaccine repetitive therapy was tested, either alone, or combined with chemotherapy. 37 patients (31 Stage IV [M1a(6), b(7), c(18)] and 6 Stage IIIc) were studied using intra-dermal VMCL vaccine therapy. If disease progressed, vaccine was continued with standard chemotherapy (DTIC and/or Fotemustine). Overall survival was assessed and clinical responses were also recorded. From vaccine commencement, median overall follow-up was 10 months. Survivals ranged from 4 to 73 months. Median (mean) overall survival was 10 (23.5) months; overall survival at 1, 2 and 3 years was $40.5 \%, 21.6 \%$ and $10.8 \%$ respectively. CR and PR occurred in 18.9\% (7) and $18.9 \%$ (7) of patients; these were durable for up to 6.1 years in 4 patients. Stable disease was noted in a further $17 \mathrm{pa-}$ tients (45.9\%). In 6 patients (16.2\%) no response to therapy was apparent. Repeated vaccinations with or without chemotherapy produced strong, durable clinical responses with overall survival $>23$ months occurring in nearly 25\% of advanced melanoma patients. The overall disease control rate $(C R, P R$ and $S D)$ was $83.7 \%$, including CR in very advanced cases. These results, in a largely unselected population of advanced metastatic melanoma patients, compare very favourably with other regimens, and notably were associated with minimal, if any, toxicity. Further analysis of this approach appears warranted.
\end{abstract}

Keywords: Vaccine Therapy, Combined Immuno-Chemotherapy, Repetitive Dosing, Advanced Melanoma, Clinical Re sponses, Prolonged Survival

\section{Introduction}

Current therapies for advanced disseminated melanoma or locally advanced disease remain seriously inadequate with typically poor clinical responses, high failure rates even when responses do occur, and options for any subsequent therapy are severely limited. Immunotherapy using vaccines has been used previously, but most studies have not persisted with continued vaccinations when disease progression has occurred, and especially when chemotherapy is administered, typically using either Dacarbazine (DTIC) or Fotemustine, which are considered as the standard treatment agents. These standard chemotherapy agents are often regarded as essentially palliative for ameliorating symptoms from metastases, but to our knowledge have seldom been tested with concurrent vaccine or other immunotherapies, apart from the interferons. Despite trials and comparisons with a variety of other chemotherapeutic and biologic agents and combinations of agents, little improvement has been possible upon standard agents. However, progression free survivals are usually less than 2 months with median overall survival from 6-9 months, with any form of systemic therapy. Percent survivals at 1-year in response to DTIC and a variety of agents is approximately $25 \%$ [1], however this reduces substantially to $<2 \%$ at 2-5 years [2]. Immunotherapy with dendritic cell vaccines has been effective in a number of small studies, but results have 
generally not justified the complex and expensive production procedures. Immunotherapy with traditional vaccines has received limited evaluation, and those available have results at least as effective as those with DTIC (reviewed by Hersey 2004) [3]. Surgery for isolated metastases may be an option in highly selected patients with localised Stage IV or Stage IIIc melanoma, but this option is usually unavailable or ineffective for treatment of widespread metastatic disease.

Vaccinia Melanoma Cell Lysate (VMCL) vaccine therapy, consisting of a vaccinia virus lysed, allogeneic melanoma cell line, has been previously described $[4,5]$ and was used in a previous Australian randomised clinical trial for earlier-stage, completely resected high-risk melanoma [6]. Although no statistically apparent overall survival benefit ( $p=0.068$ ) could be shown for the VMCL vaccine treated group over the untreated controls in Stage IIb and III melanoma patients, the survival of vaccine treated patients remained above that of the non-treated patients, indicating a possible 'positive effect' in some patients. The VMCL vaccine was shown to be very safe and demonstrated low or no toxicity in over 700 patients given the vaccine for 2 years [6]. However, no formal studies had been done in patients with residual advanced disease, so a pilot study was done in patients with advanced surgically non-resectable stage IV/ IIIc metastatic melanoma (some after prior chemotherapy), to examine whether immunotherapy with this vaccine may be of benefit in this patient group; and whether combination with DTIC or Fotemustine may further improve its efficacy. The main aim in the study was to investigate the feasibility of use of sustained, repeated dosing of the VMCL vaccine for potential therapy in patients with advanced unresectable melanoma to observe if any effect appeared to be present, and if disease progression occurred, vaccine would be continued during added chemotherapy. Endpoints were primarily measurement of overall survival, but morbidity and toxicity were also recorded, and in addition response rates were noted secondarily.

\section{Methods}

\subsection{Patient Characteristics}

The median age of the thirty-seven [37] patients enrolled in the studies was 59 years, with a mean age of 61 and age range of 35 to 97 years. All patients were evaluable for follow-up from 4 to 73 months after commencement of vaccine therapy, with a mean of 23.5 months follow-up and a median 10 months. No patients were lost to follow-up.

All patients had either advanced non-surgically re- sectable AJCC 1) Stage IV disease or 2) extensive multiple in-transit metastatic stage IIIc disease. In these studies, $67.5 \%$ (25) of patients had extensive disease, with involvement of internal organs; the remainder having advanced non-resectable subcutaneous metastatic disease of the limb or trunk (Table 1).

\subsection{Treatment Type}

Vaccine therapy was administered alone in 18 (48.6\%) patients and Vaccine was combined and continued with chemotherapy, typically with either DTIC, Fotemustine or both sequentially in 19 (51.4\%) of the 37 patients. 4 of the chemotherapy patients received Isolated Limb Infusion (ILI) chemotherapy using melphalan $(7.5 \mathrm{mg}$ per litre of limb tissue) and Actinomycin-D (50.0 $\mu$ g per litre of limb tissue) and 2 patients received only 1 dose of chemotherapy, terminated due to side-effects. CR's occurred in 7 patients: These occurred in 4 patients treated with VMCL vaccine alone, 2 patients treated with prior ILI chemotherapy and 1 patient treated with systemic chemotherapy after commencement of the vaccine.

\subsection{Patients and Inclusion/Exclusion Criteria}

Informed patient consent, Human Ethics Committee approval and trial registration with the Australian Clinical Trials Registry [ACTRN12605000425695] was obtained. Patient withdrawal could occur at any stage, and data

Table 1. Patient baseline characteristics.

\begin{tabular}{|c|c|c|c|c|c|}
\hline Parameter & & & $\begin{array}{l}\text { VMCL Treated } \\
\qquad(\mathrm{n}=37)\end{array}$ & & \\
\hline \multicolumn{6}{|l|}{$\begin{array}{l}\text { Age } \\
\text { (years) }\end{array}$} \\
\hline Mean & & & 61 & & \\
\hline Median & & & 59 & & \\
\hline Range & & & $35-97$ & & \\
\hline$<55$ & & & 14 & & \\
\hline $55-<65$ & & & 8 & & \\
\hline $65^{+}$ & & & 15 & & \\
\hline \multicolumn{6}{|l|}{ Gender } \\
\hline Male & & & 19 & & \\
\hline Female & & & 18 & & \\
\hline \multirow{4}{*}{$\begin{array}{c}\text { Perform- } \\
\text { ance Status }\end{array}$} & ECOG & & & UICC & \\
\hline & 0 & 34 & & 0 & 34 \\
\hline & 1 & 2 & & 1 & 1 \\
\hline & 2 & 1 & & 2 & 2 \\
\hline \multicolumn{6}{|l|}{$\begin{array}{l}\text { M Classi- } \\
\text { fication }\end{array}$} \\
\hline IIIC & & & 6 & & \\
\hline M1a & & & 6 & & \\
\hline M1b & & & 7 & & \\
\hline M1c & & & 18 & & \\
\hline
\end{tabular}


confidentiality was preserved. ECOG and UICC quality of life/functional status scores were measured. Ophthalmological testing (for possible melanoma associated retinopathy/iritis) and CT scans of the head, chest and abdomen were done at entry and repeated each 6 and 3 months, respectively. In all, 37 stage IV/ IIIc melanoma patients were enrolled in these studies for the primary aim (see statistical information). Inclusion Criteria: Patients over 18 years; with ECOG 0-2; evaluable metastases; primary cutaneous melanoma; advanced non-surgically resectable AJCC Stage IV or Stage IIIc; tumour volume $<20 \mathrm{~cm}$ diameter or $<70 \%$ of organ replacement; +/- post-surgical treatment of brain metastases; able to give informed consent. Exclusion Criteria were presence of another primary invasive cancer (not BCC, SCC or resected in-situ malignancy), untreated brain metastases, extremely extensive disease, bone metastases only, high-dose oral steroid therapy; pregnancy or lactation; severe atopia; severe cachexia; immunodeficiency, HIV, Hepatitis B or C positive. 3 patients were screened and found to have inoperable brain metastases and were excluded. Apart from the latter, all screened patients participated in the study.

Most patients were offered therapy provided their disease was advanced and not amenable to surgical resection.

\subsection{VMCL Vaccinations}

Vaccinations were at regular 2-weekly intervals for 6 months; then monthly for 6 months. If stabilisation/ complete response was obtained, then doses were given 3-monthly thereafter. Intra-dermal injection sites were rotated between upper outer aspects of all 4 limbs, but avoided in any limb where lymph node dissection was performed, to avoid inducing any lymphoedema. $0.3 \mathrm{ml}$ of the re-suspended, sonicated, lysate was determined as safe and practical from the previous studies of VMCL vaccine [5]. Although only minimal skin reactions were observed in previous Stage II/III melanoma studies, precautionary resuscitation facilities were available and patients were observed for $0.5-1$ hour after the $1^{\text {st }}$ and $2^{\text {nd }}$ vaccinations.

\subsection{Skin DTH Testing \& Responses}

These were performed using the $1^{\text {st }}$ and again at the $4^{\text {th }}$ VMCL vaccination dose (each read-out 48 hours later; erythema and the induration responses were independently recorded in two directions perpendicular to each other; > $10 \mathrm{~mm}$ was positive) at the vaccination sites, after ensuring that no inflammatory response pre-existed.

\subsection{Chemotherapy}

If the patient showed melanoma disease-progression during vaccine therapy, the protocol allowed for addition of standard chemotherapy with either Dacarbazine (DTIC) $\left(850 \mathrm{mg} / \mathrm{m}^{2}\right.$ at 3-weekly intervals intravenously), or Fotemustine $\left(100 \mathrm{mg} / \mathrm{m}^{2}\right.$ weekly intravenously for 3 weeks, followed by a 5 week break, then 3 weekly thereafter). Vaccinations were maintained at 2-weekly intervals over the chemotherapy period and during breaks in chemotherapy.

\subsection{Clinical End-points}

Primary End-point

Overall survival was the primary end-point assessed by survival in months from the time of commencement of vaccination to the date of analysis or death of the patient.

\section{Secondary End-points}

1) Toxicity and tolerability, in terms of local or systemic reactions, was examined with each vaccination and recorded.

2) Tumour response rates were also recorded, assessed by the WHO [7] criteria. Observable subcutaneous lesions were assessed using two perpendicular direct size measurements using calipers or a ruler, and internal metastases were assessed using CT scans at 3-monthly intervals or as clinically otherwise determined, and where appropriate using ultrasound, MRI or Positron Emission Tomographic (PET) scans.

\subsection{Statistical Analysis}

This was performed using standard means and median calculations, Kaplan-Meier analysis and time-series analysis with the assistance of our statistician and mathematician (NB; AC). All analyses were set at a significance level of 0.05 in the initial analyses.

\section{Results Clinical Outcomes}

\subsection{Primary Endpoint-Overall Survival}

Statistical analysis was performed on data collected to the end of 2006, with a median overall follow-up at this time (to either death or date of analysis) of 10 months. Overall survival for all 37 patients ranged from ranged from 4 to 73 months with a mean survival of 23.5 months and median survival of 10 months. Nine (9) of the 37 (24.3\%) patients survived greater than 23 months, ranging from $1.9-6.1$ years. The characteristics of these 9 patients are shown in Table 2. Organ and/ or lymph node metastases in 7 of 9 patients (78\%), and multiple subcutaneous metastases alone were present in 2 of 9 


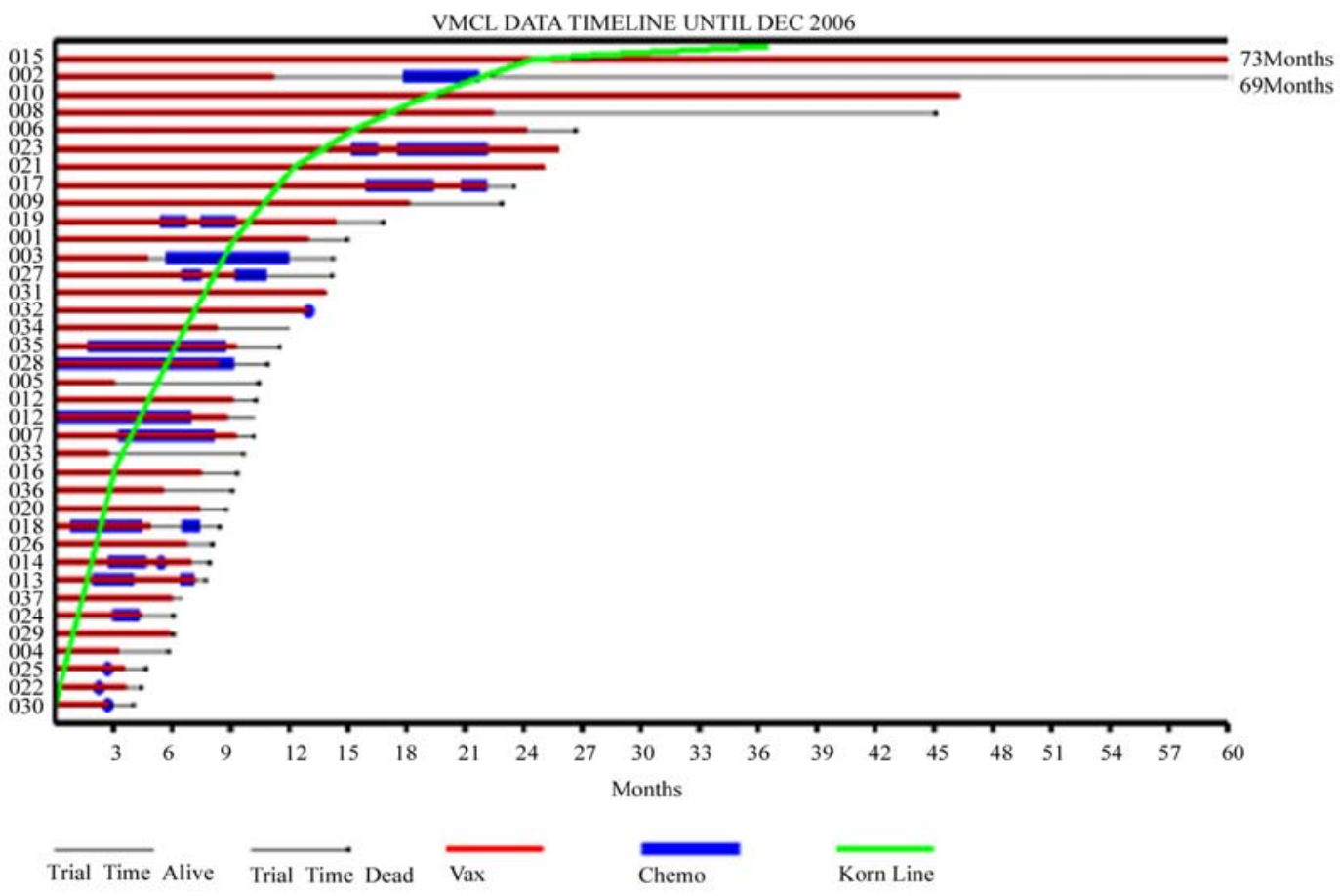

Figure 1. Bar-chart for the 37 patients indicating the survival duration time-lines; showing also the period of vaccination (red), and where used chemotherapy (blue), for each patient. (The green line indicates the comparative survival line calculated from the Korn 2008 paper data ${ }^{1}$ ).

patients (22\%).

For the entire group, at the end of the survey period 9 (24.3\%) patients were alive and well, and 28 (75.7\%) had died. The median survival time of the 9 patients still alive was 25 months from vaccine commencement, and in 5 of these patients the median survival time was 45 months. The survival times for the individual 37 patients along with the treatments given and the timing of these treatments are shown as a bar-chart in Figure 1.

Nearly half the patients (15) survived beyond 12 months and 9 patients survived for longer than 23 months. More detailed description of the patients surviving to the 2-year time-point or beyond is given in Table 3. This showed that 3 had M1c, 1 M1b and 5 M1a stage melanoma and most had failed prior radiotherapy (1), surgical metastatectomy (6), biological therapy (2) and ILI chemotherapy (1), two had received no prior therapy.

A Kaplan-Meier analysis was performed which demonstrated an overall survival at 1, 2, and 5 years was 40.5\%, 21.6\% and $10.8 \%$ respectively. These survivals compare vary favourably with the 1- and 2-year survivals of $25 \%$ and $10 \%$ [1] and $30 \%$ and $11 \%$ [2] respectively, in previous studies [1,2,8; see Table 3]. 5-year survivals are uniformly low at $<2 \%$ in most studies.

\subsection{Duration of Survival}

The survival times of patients from the time of commencement of vaccination until either death, or the time of evaluation were analyzed by stratification into 6-

Table 2. Clinical outcomes for patients surviving $>$ or $=23$ months including survival time in months, disease sites and current status.

\begin{tabular}{|c|c|c|c|c|}
\hline $\begin{array}{r}\text { Survival } \\
\text { (Months) }\end{array}$ & ID & Sites & $\begin{array}{c}\text { Past } \\
\text { Tx }\end{array}$ & Status \\
\hline 73 & 015 & s/c; LN & S & $\begin{array}{l}\text { Alive; Fully } \\
\text { functional }\end{array}$ \\
\hline 69 & 002 & lung; LN & S, B & $\begin{array}{l}\text { AWD; Fully } \\
\text { functional }\end{array}$ \\
\hline 45 & 010 & s/c; LN & S, ILI & $\begin{array}{l}\text { Alive; Fully } \\
\text { functional }\end{array}$ \\
\hline 44 & 008 & $\mathrm{~s} / \mathrm{C}$ & & $\begin{array}{c}\text { Alive; Fully } \\
\text { functional }\end{array}$ \\
\hline 28 & 021 & s/c; LN & $\mathrm{S}$ & $\begin{array}{l}\text { AWD; Fully } \\
\text { functional }\end{array}$ \\
\hline 26 & 006 & $\mathrm{~s} / \mathrm{C}$ & S & Died \\
\hline 25 & 023 & $\begin{array}{l}\text { GB; s/c; lung; } \\
\text { LN }\end{array}$ & S, B & $\begin{array}{c}\text { Alive; Fully } \\
\text { functional }\end{array}$ \\
\hline 24 & 017 & $\begin{array}{l}\text { lung; liver; } \\
\text { spleen }\end{array}$ & none & Died \\
\hline 23 & 009 & bone; s/c; lung & $S, R$ & Died \\
\hline
\end{tabular}




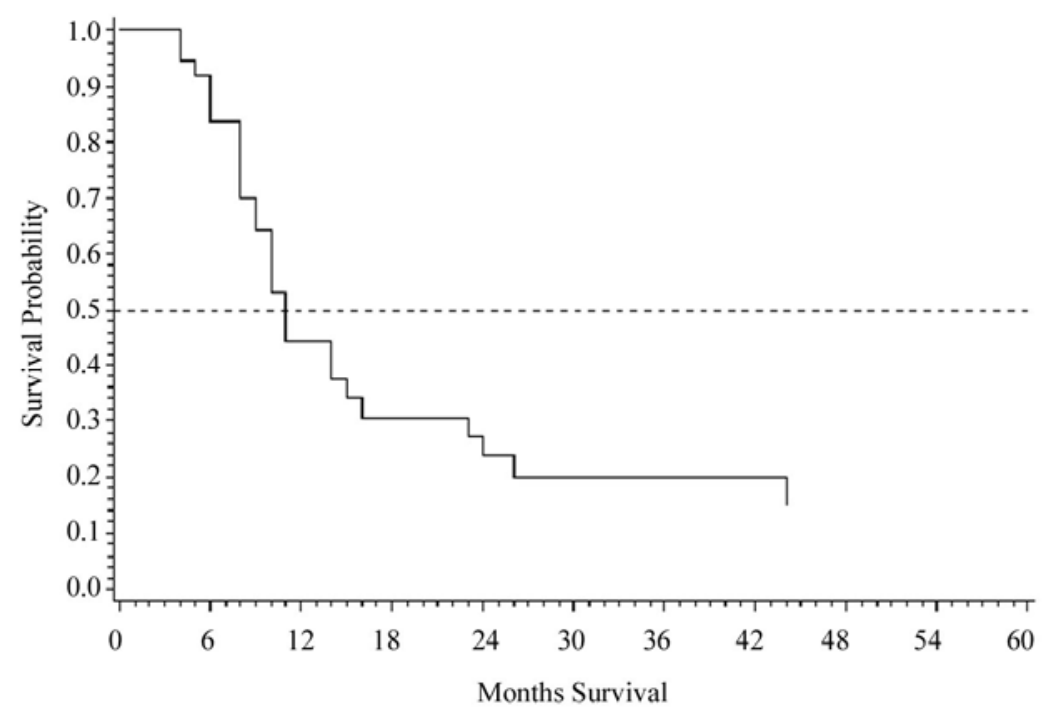

Figure 2. Kaplan-Meier survival curve for the VMCL \pm Chemotherapy Treated Patients $(\mathrm{n}=37)$.

monthly survival time groups for convenience and ease of evaluation. Survival < 6 Month was 3 patients (8.1\%); 6-12 Months 19 patients (51.4\%); 12-18 Months 6 patients (16.2\%); > 18 Months 9 patients (24.3\%); and > 44 Months 4 patients (10.8\%). Cumulative Survival, from commencement of vaccination to either death or analysis was: < 6 Months 3 patients (8.1\%); > 6 Months 34 patients (91.9\%); > 12 Months 15 patients (40.5\%); > 18 Months 9 patients (24.3\%); > 44 Months 4 patients (10.8\%).

\subsection{Clinical Disease Responses}

Clinical responses were considered as secondary end-points, as these were related to, but separate from, overall survival. This was especially so for CRs, as ex pected, but PRs were also observed to be associated with marked clinical melanoma regression (Figure 3), devel-

Table 3. Summary of Overall Survival and Complete Response comparisons for VMCL ( \pm Chemotherapy) Trial treated patients compared with standard chemo-therapy treated patients derived from recent historical data (adapted from [1,2,8]).

\begin{tabular}{ccccc}
\hline $\mathrm{N}=37$ & $\begin{array}{c}\text { Complete } \\
\text { Response } \\
\text { Rate } \\
\text { Median } \\
\text { (mths) }\end{array}$ & $\begin{array}{c}\text { 1-Year } \\
\text { Overall } \\
\text { Survival }\end{array}$ & $\begin{array}{c}\text { 3-Year } \\
\text { Overall } \\
\text { Survival }\end{array}$ & $\begin{array}{c}\text { Median } \\
\text { Survival } \\
\text { Time } \\
\text { (months) }\end{array}$ \\
\hline $\begin{array}{c}\text { VMCL } \\
\text { Therapy } \\
\pm \text { Chemo- } \\
\text { therapy) }\end{array}$ & $18.9 \%$ & $40 \%$ & $10.8 \%$ & $\begin{array}{c}10(23.5 \\
\text { mean) }\end{array}$ \\
$\begin{array}{c}\text { Standard } \\
\text { Therapies }\end{array}$ & $0.8-2.7 \%$ & $25.5-30 \%$ & $0.5-1.9 \%$ & 6.2 \\
\hline
\end{tabular}

opment of stable disease and prolonged survival in some cases. Initial complete regression (CR) of all tumors, as measured by clinical and radiological means, occurred in 18.9\% (7) of patients. Complete Durable Regression beyond 18 months (Alive $+\mathrm{CR}$ ) occurred in $10.8 \%$ (4) of patients.

Any Clinically Measurable Response was demonstrated in $83.7 \%$ (31) of patients, detected by regression of some or all melanoma lesions, either clinically for directly observable lesions, or radiologically for internal lesions. PR was demonstrated in $18.9 \%$ (7) of patients during treatment with the vaccine. Stable Disease (SD) occurred in $45.9 \%$ (17) of patients. No quantifiable response was noted in $16.2 \%$ (6) of patients, but 1 or 2 lesions in these patients showed mild erythema or minor size reduction of some lesions, which were sometimes short-lived ( $<6$ weeks), however, these were not counted as 'responses'. Progressive Disease (PD) was the cause of death in all 28 (75.7\%) who had died at the end of the study period. No patient died of non-melanoma related causes.

\subsection{Toxicity}

Anaphylaxis or allergy was not observed. No local or systemic toxicity was observed at any stage from the vaccinations during the studies. Minor skin irritation or unexplained dizziness was rare. Chemotherapy toxicity was of the same profile observed with standard chemotherapy for metastatic melanoma, and unaffected by concomitant vaccination. Side-effects of chemotherapy were generally mild with no grade 4 toxicity observed. Three patients required dose delay because of grade 3 neutropenia. Two 


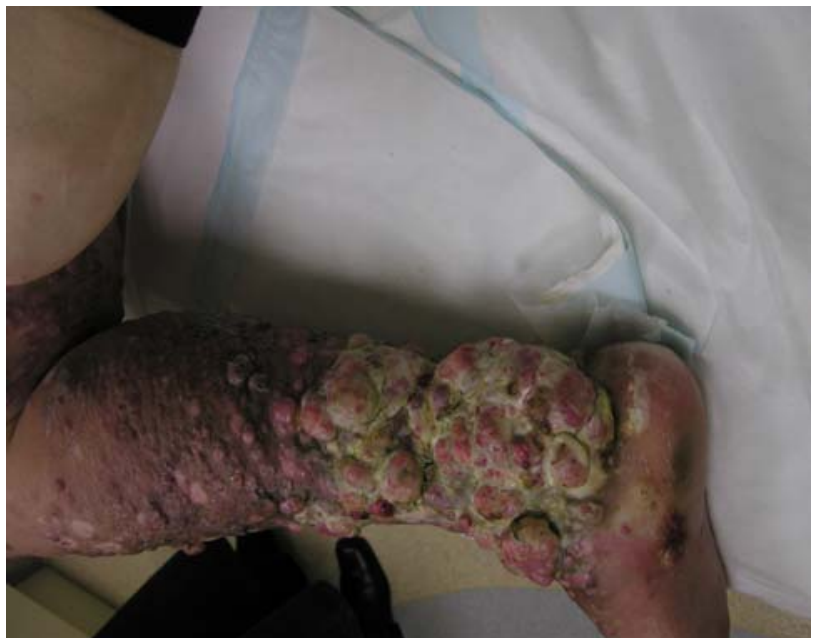

(a) August 2005

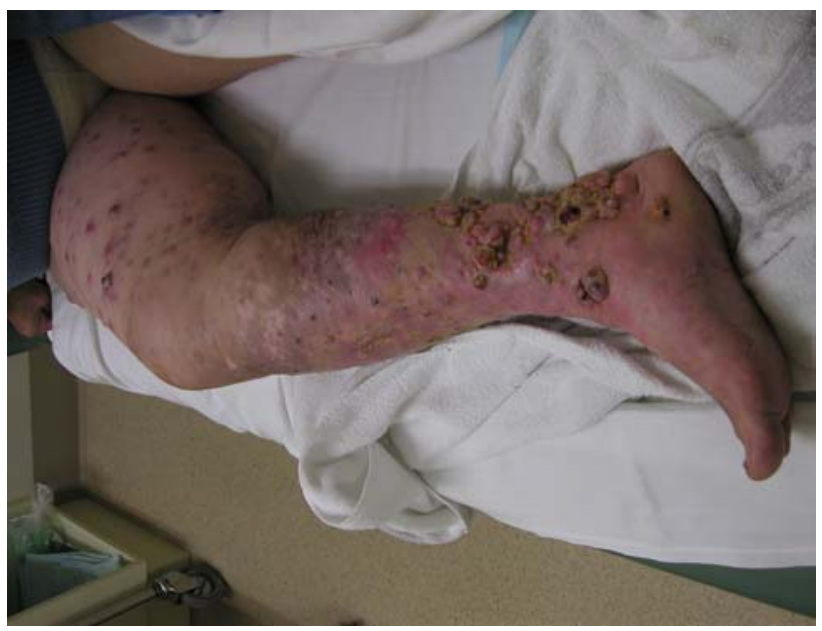

(b) December 2005

Figure 3. Patient treated with VMCL vaccine alone from August (before; a) to December 2005 (during; b). She was able to walk after the therapy and the pain, odour and self-care ability improved markedly.

patients stopped chemotherapy after one cycle due to disease progression and patient choice.

\subsection{DTH Responses}

No measurable DTH responses against VMCL were detected at either of the time-points tested in any of the 37 patients in the studies.

\subsection{Prior Failed Clinical Treatment Type}

Of the 37 patients, 20 patients (54\%) had failed previous non-surgical treatments; 14 (37.8\%) had failed previous chemotherapies (DTIC, Fotemustine, ILI chemotherapy, other biological therapies), and a further 6 (16.2\%) had failed Radiotherapy alone; refer to Table 4 and Figure 1.
Table 4. Previous (Failed) treatments (see also Figure 2).

\begin{tabular}{lc}
\hline \multicolumn{1}{c}{ Treatment } & Number \\
\hline Surgical Metastatectomy & 21 \\
Radiotherapy & 11 \\
Chemotherapy & 6 \\
ILI Chemotherapy & 4 \\
Biological Therapy & 5 \\
No Prior Therapy & 10 \\
\hline
\end{tabular}

\section{Discussion}

A possible innovative role for combined continued immuno-chemotherapy is suggested by these results in the treatment of advanced non-resectable melanoma that has (usually) failed surgery and/or other therapies. The results shown above indicate that repeated vaccine therapy, with or without concomitant standard chemotherapy was effective in inducing complete regression of advanced melanoma in nearly $20 \%$ of patients and appeared to slow melanoma growth in over $60 \%$ of patients with mean survivals of 23.5 months and 1, 2 and 3 year survivals of 40.5, 21.6 and 10.5\% respectively. An as yet undefined mechanism appears to be operating to produce these observations. The above findings compare favourably with the pooled results from the cooperative group studies reported by Korn et al. [1], which appear to have similar patient entry criteria. Our results were unexpected, as many patients had failed (often multiple) previous therapies and had extensive disease. Furthermore, one quarter of the patients (24.3\%; 9) survived over 23 months, since commencement of vaccination, and long-term durable responses of between 4 and 6 years were obtained in 4 (10.8\%) patients. Interestingly, not all of these patients had CR's to explain their clinical course with repeated vaccination. At the end of the survey period, out of the 37 patients included in the study, 9 (24.3\%) patients were alive and well, and 28 (75.7\%) had died. Almost half $(46 \% ; 17)$ of the patients were observed to develop stable disease (SD), despite having extensive disease. Somewhat predictably, overall survival was associated with clinical responses. This was especially so for CRs, but surprisingly to us, PRs were also observed to be associated with development of stable disease and prolonged survival, even in some patients with high disease burden. This suggests some true effect of repeated vaccination therapy, and possibly additional chemotherapy enhancement, although the direction of the effect (ie. which therapy is enhancing which) is presently unclear.

Although the results shown above are suggestive that immunotherapy with VMCL \pm chemotherapy may be of benefit in extending the survival of some patients with 
melanoma, it is of course appreciated that interpretation in smaller uncontrolled studies may be confounded. However, the very fact that any patients could demonstrate prolonged survivals was remarkable, and that disease progression appeared to be halted, at least for some considerable time in some patients. Although inadvertent patient selection is difficult to completely exclude, nevertheless, over $2 / 3$ of patients in the present study had much less favourable stage IV $\mathrm{M} 1 \mathrm{~b} / \mathrm{c}$ disease and included a spectrum of patients with advanced disease. The importance of stage of disease on metastatic melanoma is well reported, for example in large patient groups, 1 and 2 year survivals for M1a disease were $54 \%$ and $36 \%$ respectively compared to $35 \%$ and $18 \%$ respectively for patients with M1c disease [8]. In addition, some patients had failed/ relapsed prior to entry and some had received two or more forms of prior therapy as shown in Table 4, where surgical metastatectomy included local and organ metastatic disease resections; prior chemotherapy was standard systemic therapy and/or ILI chemotherapy; biological trial therapies included IL-18; Canvaxin; NY-ESO-1; Interferon; and P-188 therapy.

The standard use of dacarbazine (DTIC) and Fotemustine, and in some countries multi-dose regimens, have not been able to substantially alter the survival of advanced metastatic melanoma, and are regarded as essentially 'palliative'. Despite hope that interleukins, interferons and other bio-therapeutic agents would improve outcome, this has not proved to be the case, and are often limited by significant toxicity [9]. Prior studies with both DTIC and Fotemustine alone have reported response rates of $5-15 \%$, and complete response rates of $0.8-2.7 \%$ with median survival times of 5-8 months in patients with advanced melanoma. No benefit was observed with multi-agent over single agent chemotherapy [10-12]. Korn [1] in a meta-analysis of 42 phase 2 trials involving 2027 patients reported a median survival time of 6.2 months (95\% CI; 5.9-6.5 months). Percent survival at 1 -year was $25.5 \%$. Bedikian [2] reported that DTIC induced a median survival of 7.8 months and approximately a 30\% 1-year survival. Overall response was $7.5 \%$ and a $0.8 \%$ complete response rate in 395 patients. In the present studies we observed that 15 patients (40.5\%) survived 12 months or more. Our observed initial complete response rate (CR) of all clinical and radiological tumour was $18.9 \%$ (7) of patients. This remained durable beyond 18 months (Alive + CR) in $10.8 \%$ (4) of patients. Any Clinically Measurable Response was demonstrated in $83.7 \%$ (31) of patients despite many of these patients had already failed standard (multiple) therapies before entry to our study.

A potentially significant point of difference between this and previous studies is that we chose to continue regular vaccine administration for a long time period irrespective of continued tumor growth [13]. Furthermore, we also continued repeated VMCL vaccination during any chemotherapy delivery, rather than stopping vaccination entirely or even temporarily, as others have done in the past.

Whether DTIC (or fotemustine) added substantially to the benefit of immunotherapy, or the reverse was true, is not clear from this limited study, but it is our impression that combined vaccine and chemotherapy in patients with progressive disease enhanced the clinical effectiveness and importantly was capable of producing either CR or stable disease, with the net effect of prolonging overall survival. The combined additive effect of vaccine and chemotherapy might be explained by either increased antigen release due to chemotherapy, or ablation of T-regulatory cells by chemotherapy or both $[14,15]$. The timing of the administration of the VMCL Vaccine and/ or chemotherapy may be of importance in determining the clinical outcome and is the subject of on-going studies [16,17]. Previous work has shown that intra-tumoural dendritic cell (DC) numbers or poor DC activation/ presentation may play a significant role in directing the anti-tumor response and outcome [18-24], perhaps through T-cell immunosuppression [25-28]. The T-effector cells and T-regulatory cell balance in the tumour microenvironment is of likely critical importance in determining outcome in a variety of cancers [29-34] and further studies are needed to examine these aspects.

In summary, treatment with the VMCL Vaccine has proven to be safe, with no or very low toxicity, and appears to induce significant improvement in overall survival in some $40 \%$ of patients with advanced melanoma. It is capable of inducing complete regression of melanoma, and some of these are durable for long periods, extending to over 6 years. Stable disease can be induced with or without partial responses in some lesions. If disease progresses, the VMCL vaccine can be safely combined and continued with standard chemotherapy using DTIC or fotemusine, and this has added to its effectiveness in a number of patients. The tumour growth modulation producing resolution, regression, and stabilization of melanoma deposits in patients, is likely due to immunomodulation due to repeated dosing of vaccine continuously with or without concurrent chemotherapy. Further evaluation of combined vaccine and chemotherapy in a randomized trial appears warranted. 


\section{Acknowledgements}

The authors would like to thank: Nancy Briggs and Thomas Sullivan for statistical advice; Dr Andrew Coyle for mathematical advice; Drs RG Morgan, R Hamilton, D Kotasek, K Pitman, J Hokin, I Patterson for clinical patient information and participation; Martin Ashdown for reading the manuscript and helpful discussions; Professor M James, for ethical advice and support. Mr Nick Xenophon, for support and the donors and patients in every way. Dr H Koga for some of the preliminary data analysis.

\section{Declaration of Conflicts of Interest}

The authors declare no conflict of interest apart from the development of the vaccine technology. A patent is applied for and held by the respective institutions. There are currently no plans for commercialization of the vaccine.

\section{Roles of Authors}

BJC wrote the manuscript and conducted the studies with assistance in the patient and data management by A-MH; $\mathrm{PH}$ produced the vaccine and provided essential support and advice; TM managed and coordinated the chemotherapy where used and provided advice.

\section{REFERENCES}

[1] E. L. Korn, P.-Y. Liu, S. J. Lee, J.-A. W. Chapman, D. Niedzwiecki, V. J. Suman, J. Moon, V. K. Sondak, M. B. Atkins, E. A. Eisenhauer, W. Parulekar, S. N. Markovic, S. Saxman and J. M. Kirkwood, "Meta-Analysis of Phase II Cooperative Group Trials in Metastatic Stage IV Melanoma to Determine Progression-Free and Overall Survival Benchmarks for Future Phase II Trials,” Journal of Clinical Oncology, Vol. 26, No. 4, 2008, pp 527-534.

[2] A. Y. Bedikian, M. Millward, H. Pehamberger, R. Conry, M. Gore, U. Trefzer, A. C. Pavlick, R. DeConti, E. M. Hersh, P. Hersey, J. M. Kirkwood and F. G. Haluska "Bcl-2 Antisense (Oblimersen Sodium) Plus Dacarbazine in Patients with Advanced Melanoma: The Oblimersen Melanoma Study Group,” Journal of Clinical Oncology, Vol. 24, No. 29, 2006, pp 4738-4745.

[3] P. Hersey, S. W. Menzies, G. M. Halliday, T. Nguyen, M. L. Farrelly, C. DeSilva and M. Lett "Phase I/II Study of Treatment with Dendritic Cell Vaccines in Patients with Disseminated Melanoma," Cancer Immunol Immunother, Vol. 53, No. 2, 2004, pp 125-134.

[4] P. Hersey, “Active Immunotherapy with Viral Lysates of Micrometastases Following Surgical Removal of High Risk Melanoma," World Journal of Surgery, Vol. 16, 1992, pp 251-260.

[5] P. Hersey, "Evaluation of Vaccinia Viral Lysates as
Therapeutic Vaccines in the Treatment of Melanoma," Annals of the New York Academy of Sciences, Vol. 690, 1993, pp 167-177.

[6] P. Hersey, A. S. Coates, W. H. McCarthy, J. F. Thompson, R. W. Sillar, R. McLeod, P. G. Gill, B. J. Coventry, A. McMullen, H. Dillon and R. J. Simes, “Adjuvant Immunotherapy of Patients with High-Risk Melanoma Using Vaccinia Viral Lysates of Melanoma: Results of a Randomized Trial,” Journal of Clinical Oncology, Vol. 20, No. 20, 2002, pp 4181-4190.

[7] WHO Handbook for Reporting Results of Cancer Treatment. World Health Organization, 1979.

[8] C. M. Balch, A. N. Houghton, A. J. Sober and S-JS Eds., “Cutaneous Melanoma,” 5th Edition, St Louis, Missouri, Quality Medical Publishing, 2008.

[9] M. B. Atkins, "Cytokine-based Therapy and Biochemotherapy for Advanced Melanoma,” Clinical Cancer Resesrch, Vol. 12, 2006, pp 2353-2358.

[10] M. F. Avril, S. Aamdal, J. J. Grob, A. Hauschild, P. Mohr, J. J. Bonerandi, M. Weichenthal, K. Neuber, T. Bieber, K. Gilde, V. Guillem Porta, J. Fra, J. Bonneterre, P. Saïag, D. Kamanabrou, H. Pehamberger, J. Sufliarsky, J. L. Gonzalez Larriba, A. Scherrer and Y. Menu "Fotemustine Compared with Dacarbazine in Patients with Disseminated Malignant Melanoma: a Phase III Study,” Journal of Clinical Oncology, Vol. 22, No. 6, 2004, pp 1118-1125.

[11] I. Quirbt, S. Verma, T. Petrella, K. Bak, M. Charette and the members of the Melanoma Disease Site Group of Cancer Care Ontario's Program in Evidence-Based Care, "Temozolomide for the Treatment of Metastatic Melanoma” Current Oncology, Vol. 14, No. 1, 2007, pp 27-33.

[12] A. S. Mansfield and S. N. Markovic, "Novel Therapeutics for the Treatment of Metastatic Melanoma," Future Oncology, Vol. 5, No. 4, 2009, pp 543-557.

[13] E. C. Hsueh, R. Essner, L. J. Foshag, D. W. Ollila, G. Gammon, S. J. O'Day, P. D. Boasberg, S. L. Stern, Y. Xe and D. L. Morton, "Prolonged Survival after Complete Resection of Disseminated Melanoma and Active Immunotherapy with a Therapeutic Cancer Vaccine,” Journal of Clinical Oncology, Vol. 20, No. 23, 2002, pp 45494554.

[14] A. K. Nowak, B. W. Robinson and R. A. Lake, "Synergy between Chemotherapy and Immunotherapy in the Treatment of Established Murine Solid Tumors," Cancer Research, Vol. 63, No. 15, 2003, pp 4490-4496.

[15] J. Taieb, N. Chaput, N. Schartz, S. Roux, S. Novault, C. Ménard, F. Ghiringhelli, M. Terme, A. F. Carpentier, G. Darrasse-Jèze, F. Lemonnier and L. Zitvogel, "Chemoimmunotherapy of Tumors: Cyclophosphamide Synergizes with Exosome Based Vaccines,” Journal of Immunology, Vol. 176, No. 5, 2006, pp 2722-2729.

[16] B. J. Coventry, M. L. Ashdown, M. A. Quinn, S. N. Markovic, S. L. Yatomi-Clarke, A. P. Robinson, "CRP Identifies Homeostatic Immune Oscillations in Cancer Patients: A Potential Treatment Targeting Tool?” Journal of Translational Medicine, Vol. 7, 2009, pp 102-109 
[17] M. L. Ashdown and B. J. Coventry “A Matter of Time,” Australasian Science, Vol. 5, 2010, pp 18-20.

[18] B. J. Coventry, J. M. Austyn, S. Chryssidis, D. Hankins and A. Harris, "Identification and Isolation of CD1a Positive Putative Tumour Infiltrating Dendritic Cells in Human Breast Cancer,” Advances in Experimental Medicine and Biology, Vol. 417, 1997, pp 571-577.

[19] B. J. Coventry, “CD1a-Positive Putative Tumour Infiltrating Dendritic Cells in Human Breast Cancer" Anticancer Research, Vol. 19(4B), 1999, pp 3183-3187.

[20] E. E. Hillenbrand, A. M. Neville and B. J. Coventry, "Immunohistochemical Localization of CD1a-positive Putative Dendritic Cells in Human Breast Tumours," British Journal of Cancer, Vol. 79 No. 5-6, 1999, pp 940-944.

[21] B. J. Coventry, P. L. Lee, D. Gibbs and D. N. Hart, "Dendritic Cell Density and Activation Status in Human Breast Cancer - CD1a, CMRF-44, CMRF-56 and CD-83 Expression,” British Journal of Cancer, Vol. 86, No. 4, 2002, pp 546-551.

[22] A. H. Barbour and B. J. Coventry, "Dendritic Cell Density and Activation Status of Tumour Infiltrating Lymphocytes in Metastatic Human Melanoma: Possible Implications for Sentinel Node Metastases,” Melanoma Research, Vol. 13, No.3, 2003, pp 263-269.

[23] B. J. Coventry and J. Morton, “CD1a-positive Infiltrating-dendritic Cell Density and 5-year Survival from Human Breast Cancer,” British Journal of Cancer, Vol. 89, No. 3, 2003, pp 533-538.

[24] N. Chaput, G. Darrasse-Jèze, A. S. Bergot, C. Cordier, S. Ngo-Abdalla, D. Klatzmann, “Azogui O Regulatory T cells prevent CD8 T Cell Maturation by Inhibiting CD4 Th cells at Tumor Sites," Journal of Immunology, Vol. 179, No. 8, 2007, pp 4969-4978.

[25] S. Nizar, J. Copier, B. Meyer, M. Bodman-Smith, C.Galustian, D. Kumar and A. Dalgleish, "T-regulatory Cell Modulation: The Future of Cancer Immunotherapy?” Minireview British Journal of Cancer, Vol. 100, 2009, pp 1697-1703.

[26] H. Y. Wang and R. F. Wang, "Regulatory T cells and Cancer," Current Opinion in Immunology, Vol. 19, No. 2,
2007, pp. 217-223.

[27] B. J. Coventry, S. C. Weeks, S. E. Heckford, P. J. Sykes, J. Bradley and J. M. Skinner, "Lack of IL-2 Cytokine Expression despite Il-2 Messenger RNA Transcription in Tumor Infiltrating Lymphocytes in Primary Human Breast Carcinoma: Selective Expression of Early Activation Markers,” Journal of Immunology, Vol. 156, No. 9, 1996, pp 3486-3492.

[28] B. Coventry and S. Heinzel, "CD1a in Human Cancers: A New Role for an Old Molecule,” Trends in Immunology. Vol. 25, No. 5, 2004, pp 242-248.

[29] N. G. Chakraborty, S. Chattopadhyay, S. Mehrotra, A. Chhabra and B. Mukherji, "Regulatory T-cell Response and Tumor Vaccine-Induced Cytotoxic T Lymphocytes in Human Melanoma,” Human Immunology, Vol. 65, No. 8, 2004, pp 794-802.

[30] M. Ahamadzadeh and S. A. Rosenberg, "IL-2 Increases CD4+CD25+Foxp3+ Regulatory T Cells in Cancer Patients,” Blood, Vol. 107, No. 6, 2006, pp 2409-2414.

[31] D. K. Sojka, Y. H. Huang and D. J. Fowell "Mechanisms of Regulatory T-cell Suppression - a Diverse Arsenal for a Moving Target,” Review Immunology, Vol. 124, No. 1, 2008, pp. 13-22.

[32] G. Darrasse-Jèze, A. S. Bergot, A. Durgeau, F. Billiard, B. L. Salomon, J. L. Cohen, B. Bellier, K. Podsypanina and D. Klatzmann, "Tumor Emergence is Sensed by Self-specific CD44hi Memory Tregs that Create a Dominant Tolerogenic Environment for Tumors in Mice," Journal of Clinical Investigation, Vol. 119, No. 9, 2009, pp 2648-2662.

[33] G. Darrasse-Jèze, S. Deroubaix, H. Mouquet, D. Victora, T. Eisenreich, K. H. Yao, R. F. Masilamani, M. L. Dustin, A. Rudensky, K. Liu and M. C. Nussenzweig, "Feedback Control of Regulatory T Cell Homeostasis by Dendritic Cells in Vivo,” Journal of Experimental Medicine, Vol. 206, No. 9, 2009, pp 1853-1862.

[34] L. Zitvogel and G. Kroemer, “Anticancer Immunochemotherapy Using Adjuvants with Direct Cytotoxic Effects," Journal of Clinical Investigation, Vol. 119, No. 8, 2009, pp 2127-2130. 